British Journal of Psychiatry (1991), 159, 289-299

\title{
Correspondence
}

Editor: Ian Pullen

Contents: New genetic model of schizophrenia/ Sibling sex and bulimia nervosa/Transmissible dementias/'Beam them up, Scotty!'/The Yates' correction factor in chi-squared analyses/The strength of association/Reading about transcultural psychiatry/Early cortical tactile-evoked potentials, laterality and schizophrenia/Clozapine in patients with NMS/Outcome indicators in mental handicap/ Census-derived measures and planning/Screening of admissions to accident and emergency/What's in a name?/Anorexia nervosa in the elderly/Busipironeinduced mania: possible interaction with disulfiram/ Clinical irrelevance of HAD factor structure/A possible antiaggressive effect of cyproterone acetate.

\section{New genetic model of schizophrenia}

SIR: I enjoyed the provocative speculations of Roberts \& Claridge about the genetics of schizophrenia (Journal, April 1991, 158, 451-456). The notion that defects in different genes lead to similar phenotypes is plausible because of the known interactions between various neuronal systems in the brain. Such a possibility could be tested by investigating offspring of affected parents or affected halfsiblings of ill individuals. In each instance, the proband would be likely to inherit defects in different genes. It would be predicted that the subject would either suffer from a more severe illness than his relatives or manifest a different phenotype. Indeed, it has been suggested that more severe illnesses emerge following matings between psychotic individuals (Penrose, 1968). Unfortunately, not all such studies are in agreement (Elsaaser, 1952). I am not aware of any studies involving half-siblings.

The concepts of 'leaky mutations' and threshold requirements of gene products for expression of phenotypes may explain the bewildering disagreement about the mode of inheritance of schizophrenia. The suggested continuum of severity linking schizotypy and schizophrenia also has considerable appeal. However, concepts of quantitative genetics may not be applicable here. Unlike clotting disorders or eye colour in Drosophila, the schizophrenic and schizotypal phenotypes are difficult to 'measure'. The difficulties arise not only because of disagree- ment about which features are specific to these disorders, but also because it is inherently difficult to quantify behaviour. Therefore, it may be difficult to demonstrate a gene dose-effect relationship for schizotypy and schizophrenia.

ElsAaser, G. (1952) Die Nachkommen geisteskranken Ellenpaare. New York: Stechert and Hoffner.

PEnrose, L. S. (1968) Critical survey of schizophrenia genetics. In Modern Perspectives in World Psychiatry (ed. J. G. Howells). Edinburgh: Oliver and Boyd.

\section{Schizophrenia Module}

VISHWAJIT L. NimgAONKAR

Western Psychiatric Institute and Clinic

3811 O'Hara Street

Pittsburgh, PA 15208, USA

SIR: The informative article of Roberts \& Claridge (Journal, April 1991, 158, 451-456) begs the question as to how we can conceptualise schizophrenia, and thus whether analogy with, for example, blood clotting mechanisms can be sustained.

It seems likely that the biological basis of schizophrenia is a vulnerable conformation of a part of the brain, established during brain development. What do we know about the likely involvement of genetically coded polypeptides in this?

Like other parts of the body, brain development is unlikely to be the consequence of a genetically coded series of events: rather, it will be a cascade of cellular differentiation mediated by peptide regulatory factors (Slack, 1989), whose (genetically coded) production will be switched on and off by the appearance of structures related in anatomy or in time. In addition, we know from the work of Hubel and Weisel that environmental factors can influence the final structure of neural pathways (Weisel, 1982). This is probably mediated by stimulating the development of certain neural buds, and not of others - again, a possible place for the activity of peptide growth activators.

In this model, 'the gene for schizophrenia' could code for a peptide associated with the migration of neural elements. But given the spreading network of interactions during growth and development of the brain, it is not possible to construct an explanation of 
its inheritance on the supposition that 'schizophrenia results from defects in a pathway $a \rightarrow b \rightarrow c \rightarrow$ brain function'. The timing of, and the substrate for, the activity of such a gene are subject to a myriad of possible modulations, from the coincidental development of the rest of the organism, and from environmental factors. The final expression of the gene as schizophrenia could depend upon this quite as much as the details of the inheritance of the gene itself.

SLACK, J. M. W. (1989) Peptide regulatory factors in embryonic development. Lancet, $i, 1312$.

WIEsEL, T.N. (1982) The postnatal development of the visual cortex and the influence of the environment (Nobel Lecture). Bioscience Reports, 2, 351-377.

Bridgewater Hospital

Chris Holman

Eccles

Manchester M30 ORL

SIR: We were interested to read the paper by Roberts \& Claridge (Journal, April 1991, 158, 451-456) and appreciate the new approach in giving a genetic model with a dimensional view of schizophrenia. The authors have given interesting analogies of eye colour in Drosophila, and thrombin formation. Most of the ideas were centred around the percentage production of active protein and the examples were towards less than normal amounts of the resulting proteins.

They also gave another example wherein it is supposed that schizophrenia results from defects in a pathway of brain function. A complete mutation in the gene controlling a step of this pathway would result in a phenotype, the failure of development of brain function being manifest as schizophrenia.

We agree with this proposition, but want to look at it from another angle. Instead of postulating that the altered genes on one or more chromosomes produce an end effect of reduction in the amount of protein (for example an enzyme of a pathway), we can say that the end result of the mutation on a single gene acting on its own or the mutations on several genes acting in union would cause an increase rather than a decrease in the amount of protein (again an enzyme). We can cite the example of acute intermittent porphyria where the activity of the hepatic delta-amino laevulinic acid synthetase is increased several fold (Dewar, 1988). Another example is Huntington's chorea in which it has been shown that there are increased dopamine concentrations in parts of basal ganglia (Gelder et al, 1989). This possibility of increase in protein would be in conformity with the dopamine hypothesis where the dopamine-like effect is supposed to be increased. What we mean to say is that, under the given circumstances, it is also possible that the pathology can be explained by overproduction of proteins - say to $200 \%$ (not only to less than $40 \%$ as stated in the paper). So in this example, if $200 \%$ explains development of schizophrenia, $150 \%$ may explain a schizotypal state. The authors gave the example of thrombin formation and cited haemophilia as a pathological entity resulting from a block of any step in the pathway. We feel that hypercoagulability is a suitable example that can be given from our view of increased protein formation. As we have already mentioned, this approach seems to be much closer to the dopamine hypothesis.

Dewar, A. J. (1988) Companion to Psychiatric Studies, 4th edn (eds R. E. Kendell \& A. K. Zeally). Edinburgh: Churchill Livingstone. Gelder, M., GATH, D. \& MAYOU, R. (1989) Oxford Textbook of Psychiatry, 2nd edn. Oxford: Oxford Medical Publications.

RAMAMOHAN VeluRI ANGela MOHAN

St Brendan's Hospital Dublin 7, Ireland

Royal College of Surgeons in Ireland

V. T. GREENE Dublin 2

\section{Sibling sex and bulimia nervosa}

SIR: Lacey et al s data on the siblings of patients with bulimia nervosa (Journal, April 1991, 158, 491-494) led them to conclude that all-female sibships were significantly over-represented. This conclusion is based on dubious statistical reasoning. Only female bulimics were included in the study, and the authors have underestimated the impact that this will have had on the likelihood of all-female sibships. For example, in the case of a sibship of two (i.e. the female proband and just one brother or sister), the authors claim that the expected proportion of all-girl sibships is one-third. This is a surprising claim since having a brother and having a sister should be equally likely (provided one ignores the slight excess of boys in the general population), leading to an expected proportion of one half. The authors' expectation that chance alone will result in bulimic women having strikingly more brothers than sisters seems no better founded than the expectation that normal women will typically have more brothers than sisters, or that normal men will typically have more sisters than brothers.

Department of Child and Adolescent Psychiatry

R. GoOdman

Institute of Psychiatry

London SE5 8AF 